\title{
Spectral and Spatial Resolution of Semiconductor Detectors in Medical X- and Gamma Ray Imaging
}

\author{
B. J. Heismann, Member, IEEE, D. Henseler, D. Niederloehner, P. Hackenschmied, M. Strassburg, \\ S. Janssen and S. Wirth
}

\begin{abstract}
In X- and gamma ray based medical systems, detector performance is a key driver for diagnostic quality. Over the last years and decades, indirect conversion scintillator detectors have become the standard for many medical applications including X-ray Radiography, Computed Tomography and SPECT. Recently, direct conversion semiconductor detectors based on CdTe and CdZnTe (CZT) have come into focus, as they might offer improved or additional performance for specific applications.

In this paper we use generic physical models to compare the spatial and spectral resolution of both detector types. The spatial resolution is quantified by the Modulation Transfer Function (MTF). We find that the direct conversion of quanta leads to an approximately sinc-like MTF. In comparison, the MTF of a pixelized scintillator detector shows a mid-frequency drop due to optical signal cross-talk.

The spectral resolution of both detector types is described by the Detector Response Function (DRF). It yields the probability density $D\left(E, E^{\prime}\right)$ to measure an incoming quantum of energy $E$ as the output energy $E^{\prime}$. As a general result we find that fluorescence $X$-ray cross-talk on one hand and charge sharing and optical cross-talk on the other hand significantly influence the DRF in both detector types. We compare the two detector types in a Computed Tomography application. For an integrating scintillator detector, a Poisson excess noise is observed and quantified. For a counting semiconductor detector, we describe the signficant low-energy shift in $D\left(E, E^{\prime}\right)$. The weighting functions of a two bin counting detctor are analyzed and compared to tube-based approaches. The results are summarized to show the trade-off between spectral and spatial resolution in medical imaging with direct conversion detectors.
\end{abstract}

Index Terms-Direct conversion, spectral resolution, spatial resolution, semiconductor detector, scintillator, Detector Response Function, DRF, Modulation Transfer Function, MTF.

\section{INTRODUCTION}

$\mathbf{M}$ EDICAL imaging devices often rely on the detection of $\mathrm{X}$ - and gamma ray radiation. Single Photon Emission Tomography (SPECT), Positron Emission Tomography (PET), Computed Tomography (CT) as well as radiography and mammography are prominent examples. From the early stages on, scintillator detectors based on materials like NaI, LSO, GOS and CsI performed the first step of radiation detection. Over the last years, an increasing number of scientific and commercial activities have employed direct conversion semiconductor detectors for medical imaging. For lower X-ray energies, amorphous selenium detectors are routinely employed in mammography detectors. For higher X- and gamma ray

B. J. Heismann is with Siemens Healthcare, Forchheim, Germany, and the University of Erlangen-Nuremberg, Chair of Pattern Recognition, Erlangen, e-mail: bjoern.heismann@ siemens.com.

D. Henseler, D. Niederloehner, P- Hackenschmied, M. Strassburg, S. Janssen and S. Wirth are with Siemens Healtchcare, Germany. energies CdZnTe and CdTe have come into focus. SPECT prototypes in cardiology, scintimammography and small animal imaging have been presented, [1]-[3]. The authors report improved spectral resolution and underline the potential to perform dual-isotope imaging. For CT, direct conversion counting electronics and prototype systems have been built and evaluated, [4]-[7]. The high X-ray flux of more than $10^{8}$ quanta per $\mathrm{s}$ and $\mathrm{mm}^{2}$ is found to be a major challenge. A main reason for this is attributed to the dynamic material properties of CZT. It has been shown that defects like Te inclusions and subsequent inferior hole mobility lead to polarization in CZT detectors under medical imaging X-ray fluxes, [8]-[11]. The main mechanism is seen in the creation of a dynamic space charge in the semiconductor bulk, degrading the charge transport properties.

In this paper we evaluate the spatial and spectral resolution of indirect and direct conversion detectors. We establish a cascaded system model for the primary conversion processes of radiation into light or charges and the secondary transport processes in both cases. We assume that the detector material defects do not play a significant role. The results thus indicate an upper performance limit. Validation measurements for the indirect conversion detector are reported. As figures of merit we use the Modulation Transfer Function to quantify the spatial resolution and the Detector Response Function $D\left(E, E^{\prime}\right)$ to describe the spectral behavior. The chosen detector setup parameters reflect typical specifications of X-ray detection detectors.

\section{THEORY}

The Modulation Transfer Function $\operatorname{MTF}(f)$ is commonly used to describe the spatial resolution of pixelized detectors, [12]. It is commonly used to describe pixelated scintillator and direct conversion detectors, see e.g. [13], [14].

The Pulse Height Spectum (PHS) is routinely used to describe the spectral resolution of PET and SPECT detectors. A typical PHS for a CZT pixelized detector and a NaI Anger camera is shown in Fig. 1. For X-ray applications, a generalization to arbitrary input energies $E$ is helpful, [16]. The Detector Response Function (DRF) $D^{(i, k)}\left(E, E^{\prime}\right)$ yields a probability density to measure an incoming quantum of energy $E$ as the output energy $E^{\prime}$. Here the incoming quantum impinges on a central reference pixel, whereas the output energy is detected at the relative pixel position $(i, k)$. Fig. 2 visualizes the pixel positions. The pair $(0,0)$ marks the center position, $(1,0)$ the horizontal neighbors, $(0,1)$ the vertical neighbors, etc. 


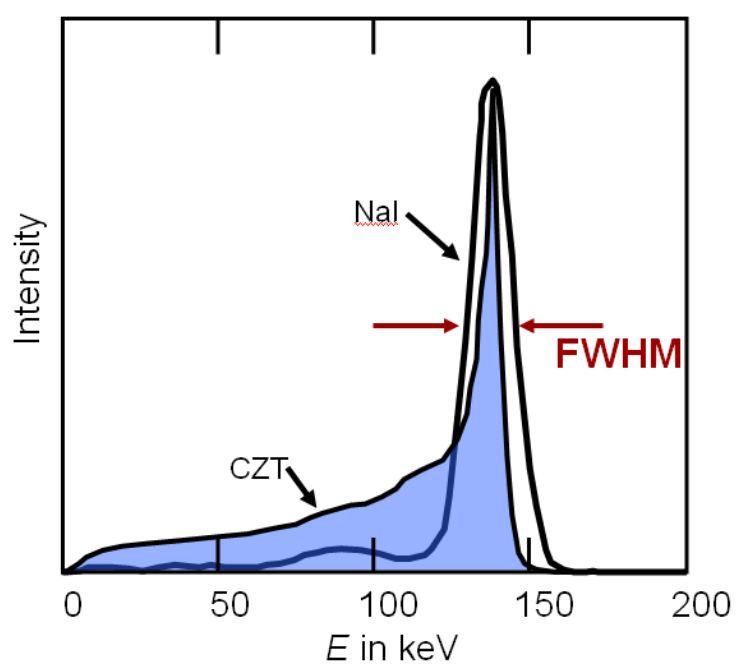

Fig. 1. Typical Pulse Height Spectrum of a NaI Anger camera and a pixelized CZT detector taken from [15].

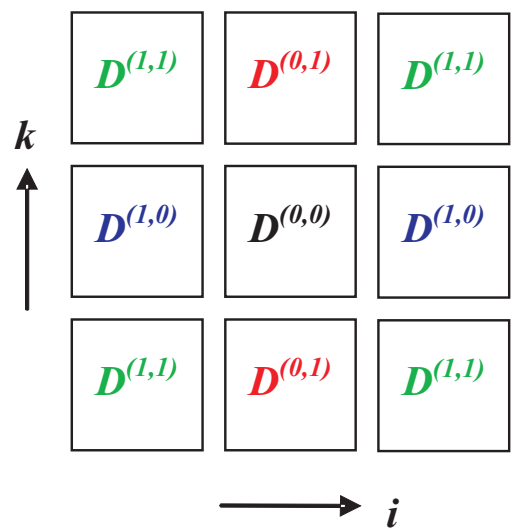

Fig. 2. Spatial indices $(i, k)$ of the Detector Response Function. The symmetry arises for pixels unaffected by border effects.

The DRF allows us to express the statistics of the microscopic signal tranport processes as a macroscopic probability function. For the purpose of this paper, we can simplify its variable dependencies. In medical imaging, the pixel-topixel variation of the projected anatomical input signal is usually in the sub-percent to percent range. This is close to a flat-field irradiation of the detector. In this case, the mean signal cross-talk between pixels is symmetrical. We realize the flat-field approximation by irradiating the detector surface homogeneously. The simplified $D\left(E, E^{\prime}\right)$ function is used to describe the results.

\section{Materials AND Methods}

Figures 3a,b show the set-up of pixelized direct and indirect conversion detectors: The scintillation detector in Fig. 3a is based on a GOS scintillator bulk material. Back-scattering $\mathrm{TiO}_{2}$ material is used to form pixels with typical dimensions of around 1 millimeter and below. A registered photosensor detects the secondary light photons at the bottom surface of each pixel ${ }^{1}$.

The main primary interaction of the detector is an absorption of an incoming X-ray quantum by a gadolinium atom. The $\mathrm{X}$-ray energxy is converted into light photons. The energy conversion rate is around 13\% [16]. Secondary light photon transport takes place. Photons which reach the photosensor contribute to the output energy signal $E^{\prime}$.

Two main physical effects in the detector have an impact on the spatial and spectral resolution in pixelized scintillator detectors: First, the primary energy deposition is not perfectly localized. For the high- $\mathrm{Z}$ atom gadolinium, absorption is governed by the photoelectric effect. This generates fluorescence escape photons with mean free path lengths in the order of several $100 \mu \mathrm{m}$. There is a substantial probability that the fluorescence quantum reaches a neighboring pixel or leaves the detector volume completely. Secondly, light transport is affected by optical cross-talk. Septa walls are designed with a limited thickness to optimize overall dose usage and light yield. As a consequence, a significant portion of the light is transfered to adjacent 'false' pixels, see Fig. 3a.

In order to describe all relevant physical transport processes we have established a Monte Carlo based simulation model, c.f. [13], [16]. Verfication measurements show that the simulation model results fit very well to spatially resolved light sensitivity and MTF measurements of CT detectors.

The direct conversion CZT scheme is shown in Figure 3b. We have chosen the common-cathode design with pixelized anodes on the bottom surface of the semiconductor bulk. Pixels are established by funnel-shaped electrical fields of several $100 \mathrm{~V} / \mathrm{mm}$.

The physics of the primary energy deposition are comparable to the indirect conversion detector. However, the deposited energy is converted to charges rather than optical photons. The holes and electrons are separated and accelerated by the electrical field. Electrical pulse signals are induced on the electrodes. Due to the small pixel effect, the main signal pulse is generated when the electrons move in the funnel-shaped electrical field region close to the anodes.

The main signal degradation mechanisms ${ }^{2}$ are comparable to indirect conversion scintillator detectors: First, fluorescence scattering takes place. Due to the lower K-edge energy, the mean free path lengths of fluorescence quanta in CZT are about $100 \mu \mathrm{m}$. The smaller the pixel size, the more fluorescence cross-talk will affect the behavior of the detector. Secondly, the charge signal transport is affected by charge sharing. The moving charge cloud also induces electrical pulses on neighboring pixels, [17], again mostly at the funnelshaped bottom part of the pixel field configuration.

We employ the following steps to model the physical processes in direct conversion detectors:

1) Primary energy deposition:

The primary energy deposition is modelled by the sim-

\footnotetext{
${ }^{1}$ Note that radiography and mammography detectors follow similar design concepts. CsI is usually employed as a scintillator. It has the advantage of providing intrinsic light guiding properties due to its needle structure. This allows for an improved pre-sampling MTF at the expense of a reduced stopping power and signal speed.

${ }^{2}$ The aforementioned defect trapping mechanisms are assumed to be negligible.
} 


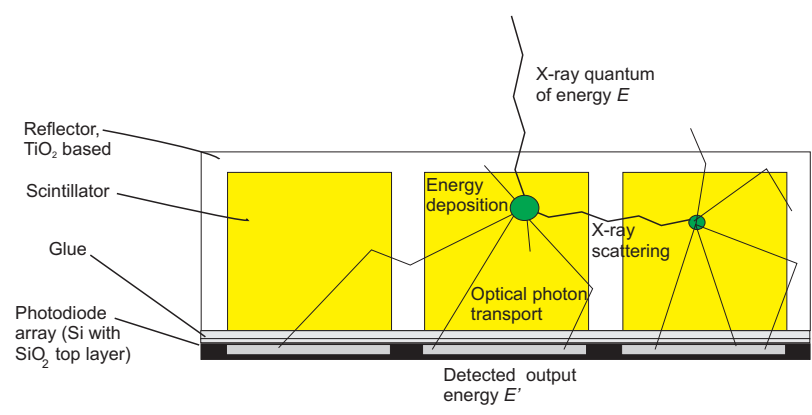

(a)

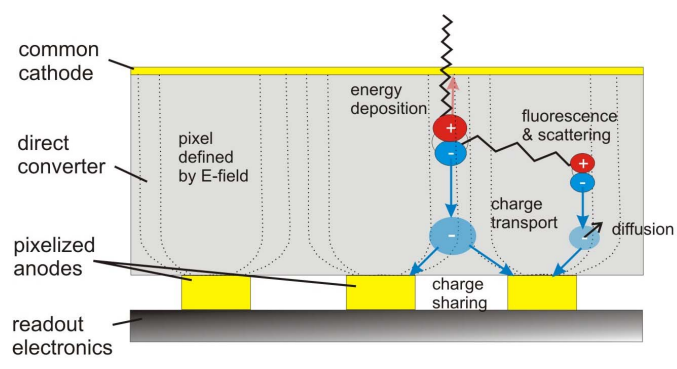

(b)

Fig. 3. (a) Scintillation detector schematics, (b) Direct conversion detector schematics.

ulation tool ROSI [18] which is based on the GEANT4 particle interaction simulation framework [19], [20].

2) Pulse generation:

A detailled charge transport model has been developed following the work of Eskin et al. [21]. A local weighting potential allows to calculate the signal pulse shape for arbitrary charge starting positions in the detector [22].

3) Electronic read-out:

We have two main electronic design schemes for direct conversion detectors, depending on the priority of spectral and spatial resolution. Spectrally resolving detectors in SPECT and PET require a precise measurement of the energy of each quantum. Due to this the anode signals are usually filtered with comparably long shaping times. The signal is integrated and digitized. High resolution detectors on the other hand address applications in mammography, radiography and Computed Tomography. The corresponding electronics employ shorter shaping times close to the primary pulse duration. The filtered pulse signals are usually detected by amplitude threshold triggering [4], [5].

For this paper we have chosen a $2 \mathrm{~mm}$ thick CZT at $700 \mathrm{~V}$ bias with a quadratic pixel size of $(450 \mu \mathrm{m})^{2}$. The electronic threshold noise was assumed as $3 \mathrm{keV}$ RMS. For this pixel size fluorescence cross-talk contributes significantly. The choice reflects mostly the high resolution case. For the GOS scintillation detector a material thickness of $1.4 \mathrm{~mm}$ and a pixel aperture of $1.2 \mathrm{~mm}$ is chosen. The spatial resolution is thus not directly comparable. We have chosen this setting to investigate if a direct conversion detector can provide improved spatial resolution at a reasonable spectral resolution.

\section{RESUltS AND Discussion}

Figure 4 shows the MTF comparison between an indirect and direct conversion detector. The straight lines in red and blue are simulated curves, whereas the corresponding dashed curves show the respective ideal sinc functions. Details on the simulations and validation measurements of the scintillation detector can be found in [13]. The indirect conversion detector shows a mid-frequency drop. This is mainly due to optical cross-talk which leads to a low-pass signal filtering in the derector. In principle the mid-frequency drop can be recovered by appropriate inverse filtering at the expense of amplified electronic noise in the signal. For high and medium flux applications, this has no major impact. The signal-to-noise ratio is mainly affected in low-flux situations like screening.

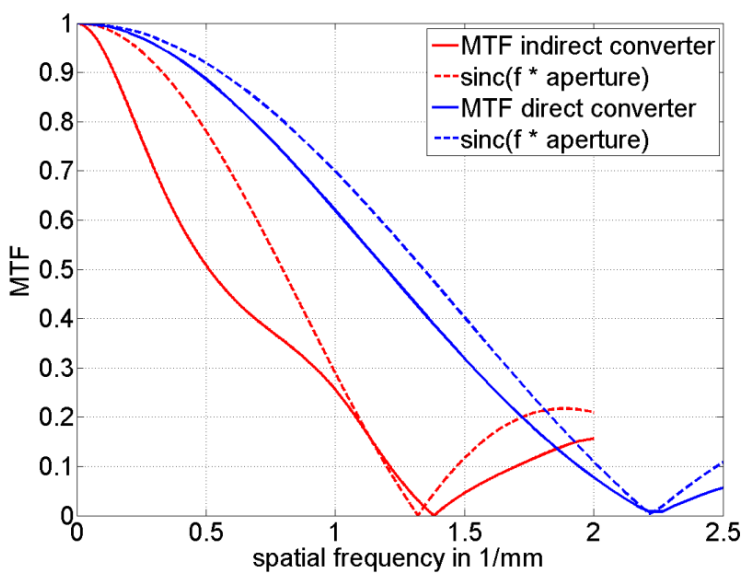

Fig. 4. Modulation Transfer Functions for indirect conversion $1.2 \mathrm{~mm}$ pixel detector (red) and direct conversion $450 \mu$ mdetector (blue). Dashed lines reflect the ideal sinc functions. The data has been reproduced from [13].

In comparison to this, the direct conversion detector is close to the ideal sinc behavior. The remaining deviations are mainly due to fluorescence escapes between adjacent pixels. Despite the fact that the pixel aperture has been more than halved, charge sharing plays only a minor role compared to the effects of optical cross-talk ${ }^{3}$.

The Detector Response Functions of the indirect and direct conversion detector set-ups are shown in Figs. 5a,b. In both cases the probabilities are normalized to 1 for each input energy $E$. This leads to the respective color codings. Below $15 \mathrm{keV}$ output energy $E^{\prime}$, the electronic noise in the counting direct conversion detector dominates the output behavior. The respective range has been omitted for better clarity.

The indirect conversion $D\left(E, E^{\prime}\right)$ in figure 5a consists of the following structures:

Up to the Gd K-edge energy $E_{K} \approx 50.2 \mathrm{keV}$, a linear branch with $E \approx E^{\prime}$ is visible. Its broadening is explained by the energy conversion gain variance. The ouput energy peak has a tail towards higher ouput energies $E^{\prime}$ for increasing

\footnotetext{
${ }^{3}$ Note that in both detector systems a small deviation in the zero frequency position is visible. This is due to the fact that fluorescence cross-talk leads to smaller signal contributions close to the pixel borders, effectively shrinking the pixel aperture.
} 


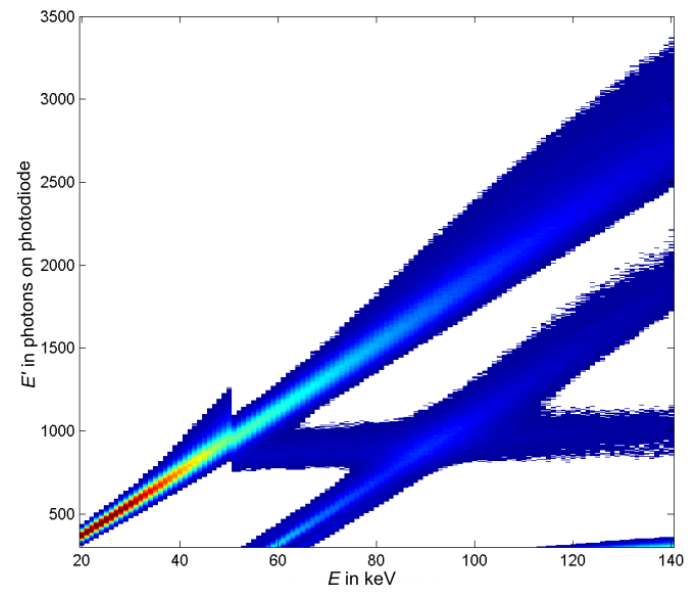

(a)

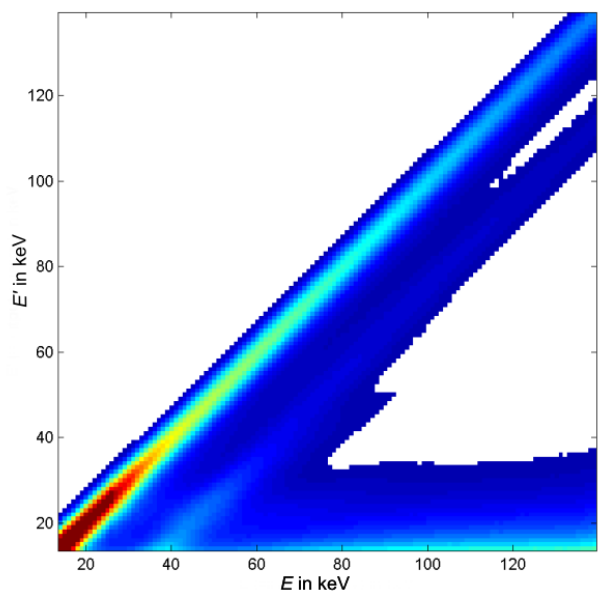

(b)

Fig. 5. Detector Response Function for (a) Indirect conversion GOS detector, (b) direct conversion CZT detector.

input energy $E$. This light tailing effect is due to the fact that the light transport yield increases with the interaction depth which in turn increases with the input energy $E$. Above the K-edge energy, a secondary branch occurs. The events are formed by an absorption of the primary energy with a fluorescence energy loss to the surroundings. The corresponding re-absorbed fluorescence events are found in the third, approximately vertical branch starting at around $50 \mathrm{keV}$ output energy. Its slight inclination is again due to the increase of the interaction depth with input energy. The overall absorption probability of the quanta is reduced with increasing input energy $E$. The low energy output events including Compton and Rayleigh scatter depositions are not shown, see [16] for a more detailed discussion of these.

The direct conversion detector $D\left(E, E^{\prime}\right)$ in Fig. $5 \mathrm{~b}$ has a more pronounced linear branch. Its stronger relative signal content is explained by the about two times higher intrinsic conversion gain of CZT and the reduced depth-dependency due to the small pixel effect ${ }^{4}$. The fluorescence branches appear at the lower $\mathrm{Cd}, \mathrm{Zn}$ and Te fluorescence energies of 23 to $28 \mathrm{keV}$. The differential branches are consequently closer to the main linear branch. Charge sharing events create a low energy tail increasing towards lower ouput energies and overlapping with the fluorescence branches.

The spectral behavior described by $D\left(E, E^{\prime}\right)$ has consequences for both detector schemes. In the following we consider the cases of an integrating indirect conversion detector and a counting direct conversion detector as prominent examples.

For integrating indirect conversion detectors it has been shown [16], that the output signal variance increase leads to a Poisson excess noise. Following the work by Rabbani et al. [23], a formula for the noise amplification has been established:

$$
f(E)=\frac{1}{\sqrt{\alpha(E)}} \frac{\mathrm{SNR}_{\text {out }}}{\mathrm{SNR}_{\text {in }}}=\frac{\left\langle E^{\prime}\right\rangle}{\sqrt{\left\langle E^{\prime}\right\rangle^{2}+\sigma^{2}\left(E^{\prime}\right)}}
$$

\footnotetext{
${ }^{4}$ Note that semiconductor defects can strongly influence this behavior.
}

with $\alpha(E)$ := quantum detection efficiency, $\left\langle E^{\prime}\right\rangle:=$ average output energy, $\sigma\left(E^{\prime}\right)$ := output energy variance.

$f(E)$ is a generalized energy-dependent Swank factor, see Fig. 6 as a result taken from [16]. The Poisson excess noise is most pronounced around the $\mathrm{K}$-edge. A noise increase of about $15 \%$ is visible. This is due to the fact that the output signal variance increases strongly beyond the K-edge. For continuous input spectra, a typical excess noise of 5\% to $10 \%$ can be estimated, depending on the input spectra and the patient attenuation.

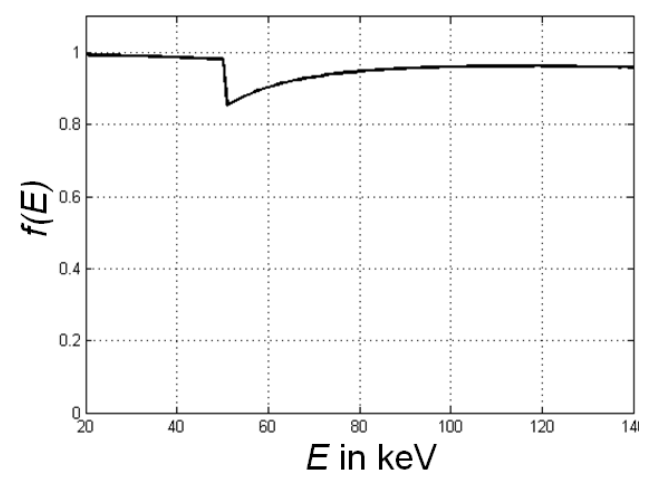

Fig. 6. Generalized Swank factor $f(E)$ reproduced from [16].

For a counting direct conversion detector we can distinguish between the full energy resolution required in SPECT or PET and the binned energy resolution required for dual-energy CT or radiography. In the case of full energy resolution, $D\left(E, E^{\prime}\right)$ contains directly the normalized PHS for specific input energies.

In the following we focus on the case of a two bin energy resolution. Like shown in Fig. 7a this is commonly achieved by using two threshold levels in the electronic read-out. The first threshold $E_{t h 1}$ discards noise events. The second threshold $E_{t h 2}$ seperates the output energy range into two separate bins. The diagonal rectangular sections mark the quanta events which are correctly assigned. The lower right region contains high energy bin primary events which are falsely assigned to 


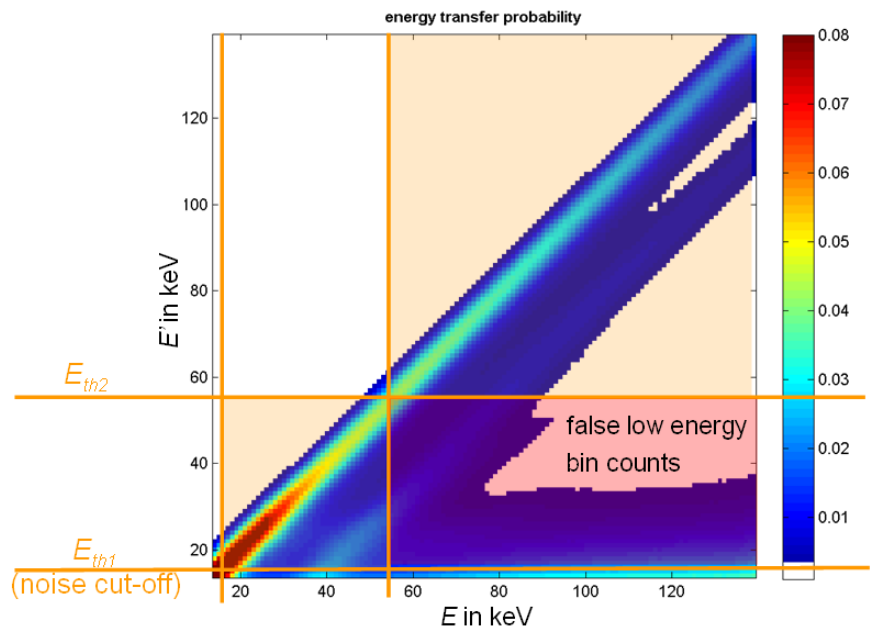

(a)

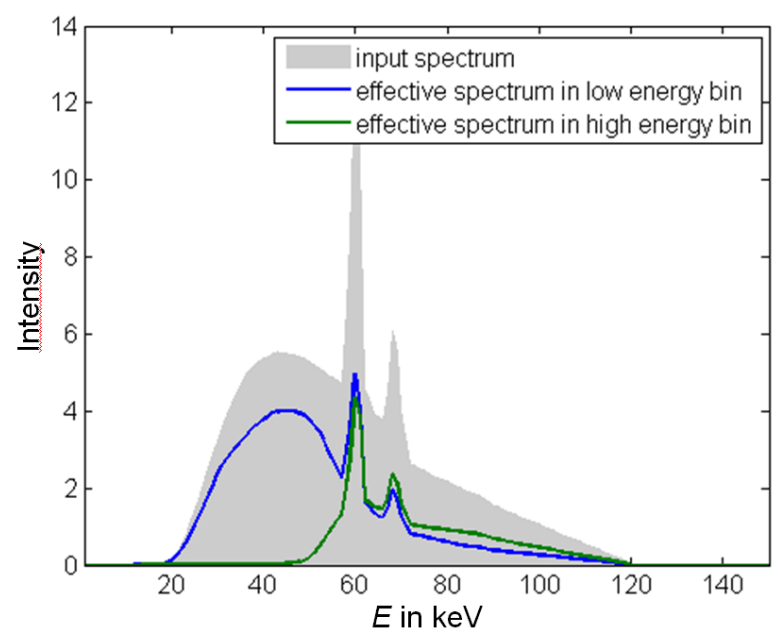

(b)

Fig. 7. (a) Schematics of energy binning for the Detector Response Function of Fig. 5a. Two energy threshold levels $E_{t h 1}=15 \mathrm{keV}, E_{t h 2}=55 \mathrm{keV}$ are used. (b) Resulting detected spectra for a $140 \mathrm{kV}$ tungsten input septrum (shaded grey).

the low energy bin.

Fig. $7 \mathrm{~b}$ shows the consequence of the low energy shift. We have assumed a $140 \mathrm{kV}$ tungsten X-ray tube input spectrum, see the shaded grey curve. The two detected spectra in the resepctive energy bins are given by the two straight curves.

Two effects are visible: First, the low energy detected spectrum loses low energy events. This is due to the fact that fluorescence events can carry away enough energy from a primary event to reduce the detected energy below the first energy threshold. Secondly, the two detected spectra overlap significantly. When we normalize both detected spectra to 1 , we obtain the system weighting functions of the two energy bins. The overlap reaches around $60 \%$ and is thus comparably larger than the 40 to $50 \%$ overlap of dual $\mathrm{kVp}$ or dual-source CT, [24]. This indicates that the dual-energy measurement capabilities of direct conversion detectors are significantly affected by low energy shift mechanisms in the Detector Response Function.

\section{CONCLUSIONS}

We have established and used physical detector models to compare the spatial and spectral resolution of indirect and direct conversion detectors. The Modulation Transfer Function $\operatorname{MTF}(f)$ and Detector Response Function $D\left(E, E^{\prime}\right)$ have been used as figures of merit.

The MTF shows a mid-frequency drop for pixelated indirect conversion scintillator detectors. This is mainly due to optical cross-talk. For direct conversion semiconductor detectors, the MTF is close to the ideal sinc function. The remaining deviations are due to fluorescence X-ray cross-talk. Charge sharing plays only a minor role for the chosen pixel size of $450 \mu \mathrm{m}$.

The Detector Response Function $D\left(E, E^{\prime}\right)$ has been used to qualify the spectral resolution of both detector types. In both cases we find a main linear branch, fluorescence branches given by the respective fluorescence energies and differential branches. The direct conversion detector has a more pronounced linear branch due to the higher average conversion gain. In the indirect conversion scintillator detector, light crosstalk and light tailing due to a dependency on absorption depth are additionally visible.

Depending on the detector readout scheme, the DRF results have different implications. Two typical cases have been investigated. In an integrating CT scintillator detector, Poisson excess noise on the measured intensity signal is observed. The generalized Swank factor shows an average noise increase of 5 to $10 \%$ depending on the input spectrum. For a counting direct conversion CT detector with energy binning the low-energy shift caused by fluorescence is significant. The weighting functions of the two bins overlap by around $60 \%$ compared to $40 \%$ to $50 \%$ in tube-based approaches.

The results indicate the trade-off between spectral and spatial resolution in direct conversion detectors. The increased spatial resolution can help to improve medical imaging applications. However, the spectral resolution of CZT detectors depends on the level of fluorescence cross-talk. For CT and radiography applications with pixel sizes in the millimter to sub-millimter range, the energy resolution is degraded significantly. Without further measures, tube-based dual-energy methods probably offer comparable or better energy resolution. For SPECT and PET, larger pixel sizes in the $2 \mathrm{~mm}$ range potentially allow to retain the good intrinsic energy resolution.

\section{ACKNOWLEDGMENTS}

The authors would like to thank Bjoern Kreisler, Thilo Michel, Juergen Durst and Michael Balda for support in detector simulations and useful discussions.

\section{REFERENCES}

[1] I. M. Blevis, M. K. O'Connor, Z. Keidar, A. Pansky, H. Altman, and J. W. Hugg, "CZT gamma camera for scintimammography," Phys. Med. Biol., vol. 21, no. Supl. 1, pp. 56-59, 2006.

[2] K. B. Parnham, S. Chowdhury, J. Li, D. J. Wagenaar, and B. E. Patt, "Second-Generation, Tri-Modality Pre-Clinical Imaging System, M0629," IEEE Nuclear Science Symposium Conference Record, 2007. 
[3] D. J. Wagenaar, J. Zhang, T. Kazules, T. Vandehei, E. Bolle, S. Chowdhury, K. Parnham, and B. E. Patt, "In Vivo Dual-Isotope SPECT Imaging with Improved Energy Resolution, MR1-3," IEEE Nuclear Science Symposium Conference Record, 2007.

[4] E. Kraft, P. Fischer, M. Karagounis, M. Koch, H. Krueger, I. Peric, N. Wermes, C. Herrmann, A. Nascetti, M. Overdick, and W. Ruetten, "Counting and Integrating Readout for Direct Conversion X-ray Imaging: Concept, Realization and First Prototype Measurements," IEEE Trans. Nucl. Science, vol. 54, no. 2, pp. 383-390, 2007.

[5] D. Moraes, J. Kaplon, and E. Nygard, "CERN DxCTA counting chip," Proceedings of the 9th International Workshop on Radiation Imaging Detectors, 2007.

[6] Y. Onishi, T. Nakashima, A. Koike, H. Morii, Y. Neo, H. Mimura, and T. Aoki, "Material Discriminated X-Ray CT by Using Conventional Microfocus X-Ray Tube and CdTe Imager, M27-2," IEEE Nuclear Science Symposium Conference Record, 2007.

[7] J. P. Schlomka, E. Roessl, R. Dorscheid, S. Dill, G. Martens, T. Istel, C. Baeumer, C. Herrmann, R. Steadman, G. Zeitler, A. Livne, and R. Proksa, "Experimental Feasibility of Multi-energy Photon Counting K-edge Imaging in Pre-clinical Computed Tomography," Phys. Med. Biol. 53, p. 40314047, 2008.

[8] S. A. Soldner, D. S. Bale, and C. Szeles, "Dynamic Lateral Polarization in CdZnTe Under High Flux X-Ray Irradiation," IEEE Trans. Nucl. Science, vol. 54, no. 5, p. 7231727, 2007.

[9] E. Bolotnikov, N. Abdul-Jabber, S. Babalola, G. S. Camarda, Y. Cui, A. Hossain, E. Jackson, H. Jackson, J. James, K. T. Kohman, A. Luryi, and R. B. James, "Effects of Te inclusions on the performance of CdZnTe radiation detectors, R27-2," IEEE Nuclear Science Symposium Conference Record, 2007.

[10] G. S. Camarda, A. E. Bolotnikov, Y. Cui, A. Hossain, S. A. Awadalla, J. Mackenzie, H. Chen, and R. B. James, "Polarization Studies of CdZnTe Detectors using Synchrotron X-ray Radiation, R27-3," IEEE Nuclear Science Symposium Conference Record, 2007.

[11] L. Abbene, S. D. Sordo, F. Fauci, G. Gerardi, A. L. Manna, G. Raso, A. Cola, E. Perillo, A. Raulo, V. Gostilo, and S. Stumbo, "Study of the spectral response of CZT multiple-electrode detectors, N24-298," IEEE Nuclear Science Symposium Conference Record, 2007.

[12] I. A. Cunningham, Applied Linear System Theory, in: Handbook of Medical Imaging, Vol. 1, J. Beutel, H. L. Kundel, and R. L. van Metter, Eds. SPIE, 2000.

[13] S. Wirth, B. J. Heismann, D. Niederloehner, L. Baetz, W. Metzger, and K. Pham-Gia, "Simulations and Measurements of the Modulation Transfer Function of Scintillator Arrays, M06-257," IEEE Nuclear Science Symposium Conference Record, 2008.

[14] T. Michel, "Energy-dependent imaging properties of the Medipix2 Xray-detector," Proceedings of Science on the 16th International Workshop on Vertex Detectors, 2007.

[15] M. N. Wernick and J. N. Aarsvold, Emission Tomography. Elsevier Academic Press, 2004.

[16] B. J. Heismann, K. Pham-Gia, W. Metzger, D. Niederloehner, and S. Wirth, "Signal transport in Computed Tomography detectors," Nucl. Inst. and Meth. in Phys. Res., A, vol. 591, 2008.

[17] T. Michel, G. Anton, M. Boehnel, J. Durst, M. Firsching, A. Korn, B. Kreisler, A. Loehr, F. Nachtrab, D. Niederloehner, F. Sukowski, and P. T. Talla, "A fundamental method to determine the signal-to-noise ratio (SNR) and detective quantum efficiency (DQE) for a photon counting pixel detector," Nucl. Inst. and Meth. in Phys. Res., A, vol. 568, pp. 799-802, 2006.

[18] J. Giersch and J. Durst, "Monte Carlo simulations in X-ray imaging," Nucl. Inst. and Meth. in Phys. Res., A, vol. 591, p. 300, 2008.

[19] S. Agostinelli et al., "G4 - a simulation toolkit," Nucl. Inst. and Meth. in Phys. Res., A, vol. 506, no. 3, pp. 250-303, July 2003.

[20] J. Allison et al., "Geant4 developments and applications," IEEE Transactions on Nuclear Science, vol. 53, no. 1, pp. 270-278, Februar 2006.

[21] J. D. Eskin, H. H. Barrett, and H. B. Barber, "Signals induced in semiconductor gamma-ray imaging detectors," J. Appl. Phys., vol. 591, p. $647,1999$.

[22] B. Kreisler, J. Durst, T. Michel, and G. Anton, "Generalised Adjoint Simulation of Induced Signals in Semiconductor X-Ray Pixel Detectors," J. Inst., no. 3, 112008.

[23] M. Rabbani, R. Shaw, and R. van Metter, "Detective quantum efficiency of imaging sytems with amplifying and scattering mechanisms," J. Opt. Soc. Am. A, vol. 4, pp. 895-901, 1987.

[24] B. J. Heismann and S. Wirth, "SNR performance comparison of DualLayer Detector and Dual-kVp spectral CT," IEEE Medical Imaging Conference Record, pp. 3280-3822, 2007. 